\title{
EVALUATION OF WAGON IMPACT TESTS BY VARIOUS MEASURING EQUIPMENT AND INFLUENCE OF IMPACTS ON CARGO STABILITY
}

This article deals with impact tests of wagons, evaluation of impacts from various measuring equipment and influence of impacts on cargo motion in the wagons when various manners of cargo securing were used. The accelerations generated during impacts were observed also by monitoring units designed for monitoring of transport and by recordings from cameras GoPro Hero. Instead of observing the influence of impacts on the cargo loaded in impacting wagons, the function of the monitoring units in a closed building with not very good GPS signal was also observed.

Keywords: Impact tests, wagon, acceleration, lashings, damages in railway transport.

\section{Introduction and description of measuring equipment}

One of the most significant risks in railway transport is the risk of damages on transported cargo due to impacts, which are generated mainly during fly or gravity shunting. Damages on cargo can be caused e.g., by its stroke on wagon's sidewalls, by drop or by shaving between pieces of cargo, between cargo and floor or sidewalls as a result of undesirable cargo movement. This risk is most significant for consignments of one or several wagons, which are not transported as block trains, because during long-haul transports these wagons can be repetitively shunted in railway hubs with cargo inside them.

UIC Loading Guidelines state that in railway transport is needed to take into account occurrence of accelerations up to $4 \mathrm{~g}$ in longitudinal direction when cargo is rigidly secured, up to $1 \mathrm{~g}$ in longitudinal direction when cargo can slide lengthways in the wagon. Accelerations up to $1 \mathrm{~g}$ in longitudinal direction should also be taken into account for wagons in block trains or those used for intermodal transport. Accelerations up to $0.5 \mathrm{~g}$ in transverse direction and up to $0.3 \mathrm{~g}$ in vertical direction are also defined in these guidelines. Such accelerations occur mainly during shunting and their duration is in general about $100 \mathrm{~ms}$ [1].

Experience from research done by Mariterm $\mathrm{AB}$ advert to a fact that number and intensity of accelerations actuating on cargo loaded in wagons are strongly influenced by type of wagons used (construction of running gear and buffers). It was found out that occurrence of accelerations exceeding trig values (0.9 $\mathrm{g}$ in longitudinal axis, $0.5 \mathrm{~g}$ in transverse and vertical axis) was rapidly different among five wagons of various types, which were transported together. During testing transport and impact tests vertical accelerations between $0.5-0.8 \mathrm{~g}$ (in few cases even up to $1 \mathrm{~g}$ ) were often measured in commonly used types of wagons with no special adjustments of either running gear or buffers [2]. We also tested wagons of common construction, which are often used in Central European Railways.

According to Table 4 of UIC Loading Guidelines the purpose of impact tests is to check whether the loading methods used and loose fastenings stand up to the longitudinal stresses exerted during railway operation. In compliance with the testing method, the wagons to be used should be fully-loaded. There should be done 2 impacts in the same direction, $\left(1^{\text {st }}\right.$ impact at $5-7 \mathrm{~km} / \mathrm{h}$ and $2^{\text {nd }}$ impact at $8-9 \mathrm{~km} / \mathrm{h}$ ) followed, without any adjustment of the load fastenings, by counter-shock (impact in opposite direction) at 8-9 km/h with these wagons, fly or gravity shunted [1].

This article deals with the impact tests of wagons, evaluation of impacts by various measuring devices, and influence of impacts on cargo motion in the wagons with three various manners of cargo securing. For these purposes 10 impact tests were performed, for which the following wagons loaded with coils of steel wire were used (Table 1):

\footnotetext{
* Jan Zamecnik, Juraj Jagelcak

Department of Road and Urban Transport, Faculty of Operation and Economics of Transport and Communications, University of Zilina, Slovakia E-mail: zamecnik@fpedas.uniza.sk
} 
Description of wagons used for the impact tests

Table 1

\begin{tabular}{|c|c|c|c|c|c|c|c|}
\hline Wagon type & $\begin{array}{c}\text { Loading } \\
\text { length }(\mathrm{m})\end{array}$ & $\begin{array}{c}\text { Length including } \\
\text { buffers }(\mathrm{m})\end{array}$ & $\begin{array}{c}\text { Wagon weight } \\
(\mathrm{kg})\end{array}$ & $\begin{array}{c}\text { Cargo weight } \\
(\mathrm{kg})\end{array}$ & $\begin{array}{c}\text { Total weight } \\
(\mathrm{kg})\end{array}$ & Floor & Buffers \\
\hline \multicolumn{7}{|c|}{ Impact wagon } \\
\hline Eas & 12.8 & 14.04 & 22500 & 52000 & 74500 & cat. A \\
\hline \multicolumn{7}{|c|}{ Tested (impacting) wagons } \\
\hline Res & 18.5 & 19.9 & 24100 & 31740 & 55840 & wooden, damp & cat. A \\
\hline Rils & 18.53 & 19.9 & 24000 & 44284 & 68284 & wooden, dry & cat. A \\
\hline
\end{tabular}

Buffers with $105 \mathrm{~mm}$ stroke are, in accordance with UIC 526-1, divided into 3 groups, where for category A energy absorption of at least $30 \mathrm{~kJ}$ is required, for category B at least 50 $\mathrm{kJ}$ and for category $\mathrm{C}$ at least $70 \mathrm{~kJ}$.

Following equipment was used for measuring of acceleration in these tests:

- device Saver 9X of the University of Zilina, placed in the middle of the tested wagon, using sample rate $1000 \mathrm{~Hz}$;

- device MoniLog EnDal Curve of CD Cargo placed in the middle of the tested wagon;

- monitoring units A1 and A2 equipped with tri-axial accelerometers, fixed on the wagon by magnetic plates, both placed close to one another on the side of impact of the impacting wagon in the same height as buffers. These units can be used for the tracking and tracing of transport (including intermodal transport) and can automatically detect the accident situations;

- monitoring units X1 and X2 equipped with tri-axial accelerometers, fixed on the wagons by magnets (magnets are their integral part). Unit X1 was placed on the impact wagon and $\mathrm{X} 2$ on the testing wagon on the side of impact. These units are other type of monitoring units used for the tracking and tracing of transport (including intermodal transport) and also can automatically detect the accident situations;

- 6-axis MotionTracking device (next as MT6) equipped with a tri-axial accelerometer and tri-axial gyroscope, the accelerometer was set to range $+/-8 \mathrm{~g}$ for purposes of these tests. The real sample rate was around $65+/-3 \mathrm{~Hz}$;

- 2 cameras GoPro HD Hero 3. Recordings from them were processed by the Tracker software and then we calculated acceleration in longitudinal axis $\mathrm{x}$ from its results. We used frame rate 120 frames per second.

All the wagons were during the impact tests loaded by coils of wire weighing about $2000 \mathrm{~kg} /$ coil. Ten impact tests were done in three series with different wagons and cargo loading:

- in the first series the wagon Res was used as tested (impacting) wagon. This series consisted of 2 impact tests in front direction (next as Res F01 and Res F02) and one test in reverse direction (Res R03);

- the Res wagon was also used in the second series, but the way of cargo securing was different. This series consisted of 2 impact tests in front direction (next as Res F04 and Res F05) and one test in reverse direction (Res R06);

- in the third series the wagon Rils was used as a tested wagon. This series consisted of 2 impact tests in front direction (Rils F07 and Rils F08) and 2 in reverse direction (Rils R09 and Rils R10), because of the low speed before shock in the test Rils R09.

The tests were done in a closed hall where the temperature was from 5 to $7{ }^{\circ} \mathrm{C}$

\section{Impact tests and their influence on loaded cargo}

The maximum recorded accelerations in the longitudinal axis $\mathrm{x}$ by particular equipment are stated in Table 2 (instead of GoPro cameras from which the average accelerations in the impact duration of 0.06 to $0.125 \mathrm{~s}$ are stated). Particular series of impact tests are highlighted by different colours.

In the first series of impact tests the Res wagon with 16 coils was used as an impacting wagon. 15 coils were loaded on the floor, one coil was placed in the upper layer in the saddle between the $8^{\text {th }}$ and $9^{\text {th }}$ coil from the front. There was a space of $60 \mathrm{~cm}$ between these coils. The coil in the upper layer was fixed with each of neighbouring coils by a polyester lashing strap with the lashing capacity of 3000 daN (Fig. 1 on the left). No blocking of the cargo was used in transverse direction. It was supposed that the friction between coils and wagon floor and an effect of pushing the windings of particular neighbouring coils one into another will be satisfying for fixation of coils in transverse direction.

After the first impact (Res F01) the coil in upper layer skipped up and all the coils moved frontwards (closer to the impact wagon). The space under the coil in the upper layer was shortened from $60 \mathrm{~cm}$ to only $5 \mathrm{~cm}$ and in the rear part of wagon the $40 \mathrm{~cm}$ long space was created. After the second impact the same situation was repeated and the coils in the front part of wagon pushed more one onto another while the space in the rear part of wagon was lengthened to $50 \mathrm{~cm}$. The third impact was done in reverse direction. The coil in the upper layer skipped up again and all the coils moved frontwards (in direction to the impact wagon). $40 \mathrm{~cm}$ long space was created between the last coil in the rear and the wagon rear wall. The space under the coil in the upper layer lengthened from 5 to $15 \mathrm{~cm}$. This manner of 
Accelerations in the $\mathrm{x}$-axis recorded in particular impact tests. All the values of accelerations are stated in $\mathrm{g}$

Table 2

\begin{tabular}{|c|c|c|c|c|c|c|c|c|c|}
\hline \multirow[t]{2}{*}{ Impact test } & \multirow{2}{*}{$\begin{array}{c}\text { Speed before } \\
\text { impact } \\
{[\mathrm{km} / \mathrm{h}]}\end{array}$} & \multicolumn{4}{|c|}{ In the place of impact } & \multicolumn{3}{|c|}{$\begin{array}{l}\text { On the floor in the middle of impacting } \\
\text { wagon }\end{array}$} & \multirow[b]{2}{*}{ GoPro Hero 3} \\
\hline & & $\mathrm{A} 1$ & $\mathrm{~A} 2$ & $\mathrm{X} 1$ & $\mathrm{X} 2$ & MT6 & Saver $9 \mathrm{X}$ & EnDal Curve & \\
\hline Res F01 & 5.76 & 1.56 & 1.68 & 1.01 & 1.34 & 1.48 & 2.24 to -2.01 & N/A & 1.57 \\
\hline Res F02 & 8.67 & 2.12 & 2.04 & 1.54 & 1.76 & 2.48 & $\mathrm{~N} / \mathrm{A}$ & N/A & 1.69 \\
\hline Res R03 & 8.89 & 2.06 & 2.08 & 1.21 & 1.31 & 1.41 & 3.30 to -2.08 & 1.82 & 1.92 \\
\hline Res F04 & 6.67 & 1.60 & 1.48 & 1.17 & 1.18 & 2.44 & 2.14 to -1.12 & N/A & 1.42 \\
\hline Res F05 & 11.08 & $0.20^{3}$ & $0.20^{3}$ & 1.40 & 1.23 & 2.06 & $\mathrm{~N} / \mathrm{A}$ & N/A & 1.84 \\
\hline Res R06 & 10.14 & 2.32 & 2.00 & 1.39 & 1.78 & 2.02 & $\mathrm{~N} / \mathrm{A}$ & 2.32 & $3.13 / 2.18^{1}$ \\
\hline Rils F07 & 6.99 & 1.44 & 1.56 & 1.11 & 1.09 & 1.60 & 0.82 to -0.71 & $\mathrm{~N} / \mathrm{A}$ & 1.37 \\
\hline Rils F08 & 8.00 & 1.72 & 1.88 & 1.45 & 0.57 & 1.16 & 2.01 to -4.33 & $\mathrm{~N} / \mathrm{A}$ & 1.61 \\
\hline Rils R09 & 5.33 & 1.16 & 1.08 & $\mathrm{~N} / \mathrm{A}$ & 0.93 & 0.95 & 0.81 to -0.72 & $\mathrm{~N} / \mathrm{A}$ & $1.67 / 0.81^{2}$ \\
\hline Rils R10 & 10.14 & 2.16 & 2.20 & 1.25 & 1.22 & 1.39 & 1.01 to -0.83 & $\mathrm{~N} / \mathrm{A}$ & 2.99 \\
\hline
\end{tabular}

cargo securing cannot be considered as satisfying because of the cargo movement after all the shocks, when in the rear part of wagon the coils can move not only in longitudinal direction but there is also a risk of their movement in transverse direction (the coils can move frontwards and rearwards, so their windings do not fit one into another and do not block particular coils against movement in transverse direction). The coil in the upper layer does not fulfil crowding-out effect.

The second series of impact tests was done with the same wagon and cargo loading distribution, but the way of securing the upper coil was different. The upper coil was secured by a loop lashing by two straps to lashing points of the wagon. After the first impact (F04) the cargo did not move. After the second impact a 5 $\mathrm{cm}$ long free space was created in the rear part of wagon and the space under the upper coil was shortened by the same length. The speed before impact was in this case higher than UIC Loading Guidelines recommend for impact tests. After the reverse impact R06 whole cargo moved frontwards to the place where had been the space created after previous impact. The space of the same length was created in the rear part of the wagon. The biggest acceleration in the longitudinal axis $\mathrm{x}$ was measured in this reverse impact by most of devices which were used (see Table 2). High peak values of acceleration were recorded also in vertical axis $z$.

In the third series of impact tests the wagon Rils was used. The wagon was loaded by 22 coils, 15 of which on the floor and 7 in the upper layer. The coils 1 to 3 and 5 to 7 were fixed always

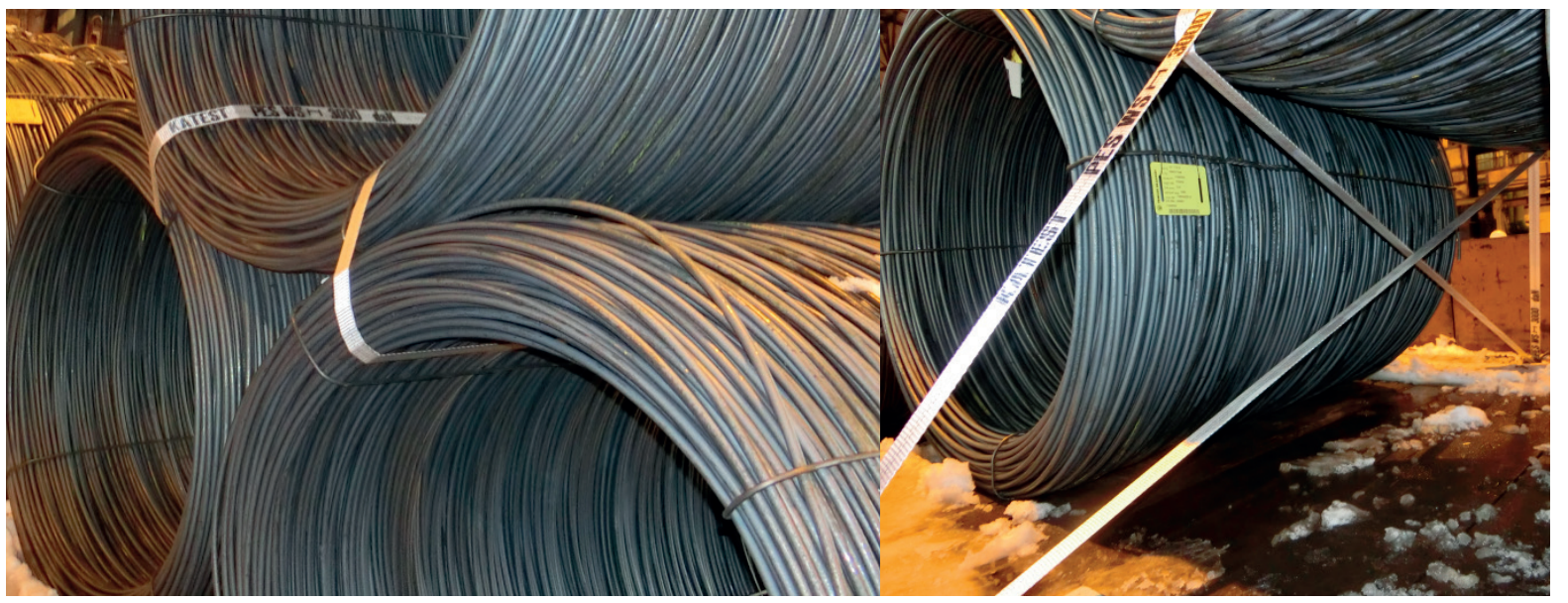

Fig. 1 Securing of the coil in the upper layer in the first series of impact tests (on the left) and in the second series of impact tests (on the right)

${ }^{1}$ Higher value was found out in duration of $0.075 \mathrm{~s}$, the lower one in duration of $0.125 \mathrm{~s}$. The impact wagon moved after this impact.

${ }^{2}$ Higher value was found out in duration of $0.075 \mathrm{~s}$, it is also possible to evaluate the deceleration as $0.81 \mathrm{~g}$ in duration of $0.2 \mathrm{~s}$.

${ }^{3}$ In these cases the monitoring units evaluated other shock instead of the shock during the impact. This shock actuated mainly in the vertical ( $\mathrm{z}$ ) axis and occured immediately before the impact, when the impacting wagon passed through an obstacle on the track. 


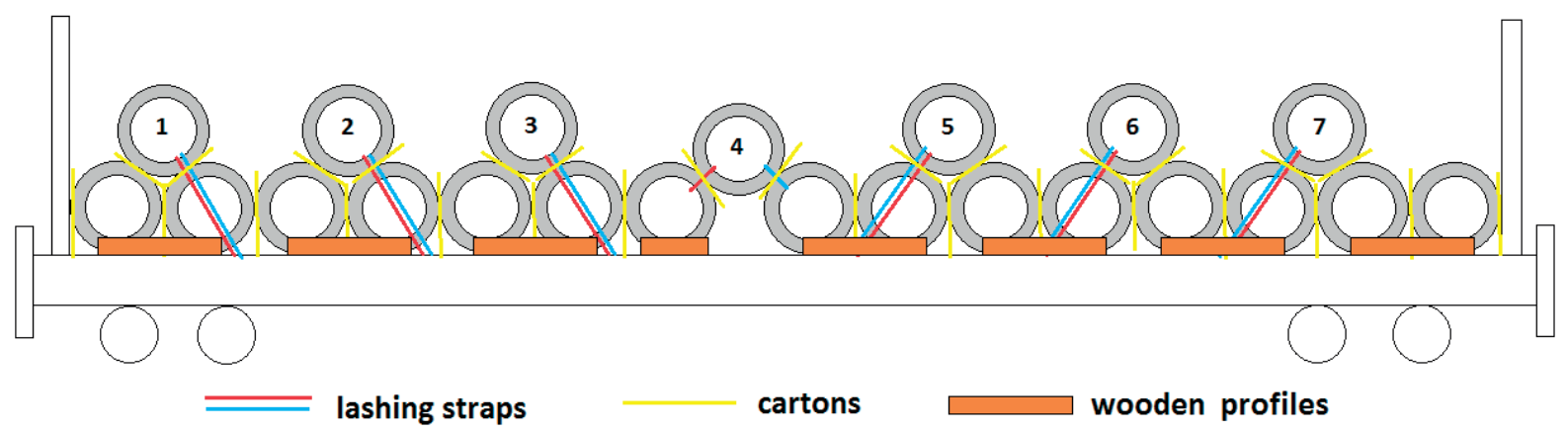

Fig. 2 Cargo loading on the wagon Rils in the third series of impact tests

by a loop lashing with two lashing straps to the lashing points of the wagon (see Figs. 2 and 3), whilst the middle-placed coil no. 4 was lashed by lashing straps only with the neighbouring coils placed on the floor and there was $60 \mathrm{~cm}$ long space under it. The neighbouring coils were separated by $5 \mathrm{~mm}$ thick cartons. The cargo was blocked against transverse movement by wooden profiles. After the first impact (F07) the coils in the upper layer skipped up but no movement of cargo was detected. The forces in lashings in the front part of the impacting wagon increased, but lashing straps in the rear part of the wagon became looser. After the second impact (F08) the $5 \mathrm{~cm}$ long space was created in the rear part of the wagon. This space was created because the coils pushed one into another and the space under coil no. 4 was not shortened. The measuring devices MT6 and Saver 9X placed in the middle of the wagon, measured high acceleration in the vertical axis in this impact ( $1.08 \mathrm{~g}$ by MT6). The speed of impacting wagon before the third impact in reverse direction (R09) was very low, so the reverse impact test was repeated. In the repeated reverse impact test R10 the cargo moved frontwards and $5 \mathrm{~cm}$ long space was created in the rear part of the wagon. Loose lashing straps in the front part of wagon (which had been its rear part in F07 and F08) became tightened and the straps in the rear part of the wagon became looser.

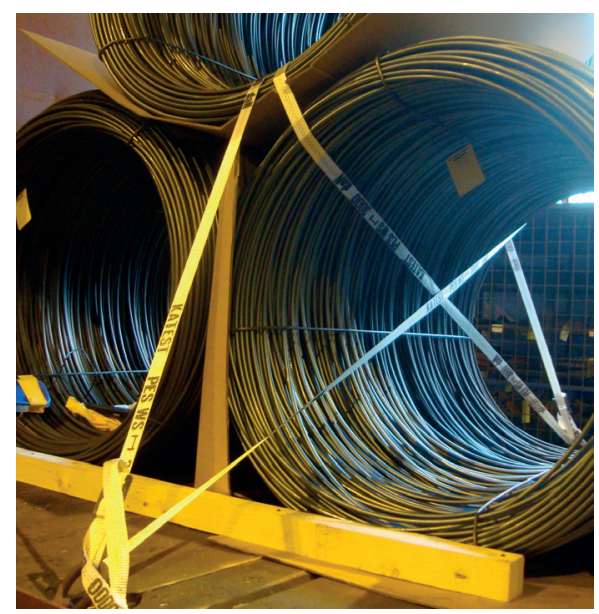

Fig. 3 Lashing on the wagon Rils in the third series of impact tests
The accelerations in the place of impact were measured by four monitoring units which are used for the monitoring of road, railway or intermodal transport and are also equipped with accelerometers with a function of accident situations detection. Units marked as A1 and A2 were placed in the same height as wagon buffers and in general these units recorded higher values of acceleration than units called X1 and X2 placed about $75 \mathrm{~cm}$ higher. Average values of accelerations in the axes $\mathrm{X}, \mathrm{y}$ and $\mathrm{z}$ from all the measuring devices, except GoPro Hero 3 cameras (the data analysis does not allow to determine the accelerations in the axes $\mathrm{y}$ and $\mathrm{z}$ ) and device Saver 9X (unsuitable data structure), are given in Table 3. Incorrect data from monitoring units A1 and A2 at impact test Res F05 are also not included to the average values. The last column states maximum values of acceleration in the vertical axis measured by MT6 (this device allowed to analyse runs of accelerations in all three axes during and after impacts). These maximum values occurred with time shift of $50-80 \mathrm{~ms}$ from occurrence of maximum values of accelerations in the longitudinal axis, as is visible in Figs. 5 and 6.

The highest accelerations were reached in the longitudinal axis $\mathrm{x}$. In several cases the impacted wagon moved after the impact, mostly in the tests Res F01, Res F02, Res F05, Res F06 and Rils F08. Average values of accelerations in the y-axis and the z-axis were recorded as about $25 \%$ and $43 \%$, respectively, of the average value of acceleration in the $x$-axis. Transverse acceleration in the $y$-axis in the three cases lightly exceeded 0.5 $\mathrm{g}$ which is the value considered for cargo securing to prevent the movement sidewards in railway transport. In general, results from these tests indicate that transverse accelerations reach higher values in the impact tests in front direction when there is no space near the frontwall of the wagon. Accelerations actuating in the vertical axis $\mathrm{z}$ in their peak values mostly exceeded $0.3 \mathrm{~g}$, as is stated in UIC Loading Guidelines, but their peak values actuated only for short-term. The device MT6 placed in the middle of tested wagons measured very high peak values of vertical acceleration in most cases, whereas in some cases it recorded two peaks, mainly in such cases when the coils skipped. Peak values in the vertical axis actuated for shorter time than that in the longitudinal axis (see Fig. 6). The problem for 
Accelerations in three axes in particular impact tests as average from various measuring equipment

Table 3

\begin{tabular}{|l|c|c|c|c|}
\hline \multirow{2}{*}{ Impact test } & \multicolumn{3}{|c|}{ Average accelerations } & \multicolumn{2}{c|}{$\begin{array}{c}\text { Maximum values of } \\
\text { vertical (z) acceleration measured by MT6 }\end{array}$} \\
\cline { 2 - 5 } & longitudinal (x) & transverse (y) & vertical (z) & 0.88 \\
\hline Res F01 & 1.44 & 0.59 & 0.80 & 1.52 \\
\hline Res F02 & 2.17 & 0.53 & 0.58 & 0.60 \\
\hline Res R03 & 1.64 & 0.26 & 0.62 & 0.95 \\
\hline Res F04 & 1.90 & 0.48 & 1.14 & 1.70 \\
\hline Res F05 & 1.69 & 0.49 & 1.00 & 2.01 \\
\hline Res R06 & 2.02 & 0.44 & 0.59 & 0.80 \\
\hline Rils F07 & 1.45 & 0.45 & 0.64 & 1.08 \\
\hline Rils F08 & 1.28 & 0.51 & 0.28 & 0.25 \\
\hline Rils R09 & 1.01 & 0.07 & 0.37 & 0.30 \\
\hline Rils R10 & 1.55 & 0.19 & 0.69 & 1.04 \\
\hline Average & 1.61 & 0.40 & & \multirow{2}{*}{0} \\
\hline
\end{tabular}

Values of speed before impact and acceleration in x-axis during impact calculated from analysis of video recordings from GoPro Hero 3 cameras

\begin{tabular}{|c|c|c|c|c|c|c|}
\hline Impact test & $\begin{array}{l}\text { Referential speed found out by } \\
\text { stopwatch }[\mathrm{km} / \mathrm{h}]\end{array}$ & $\begin{array}{l}\text { Speed - cal. } \\
\text { dist. } 1[\mathrm{~km} / \mathrm{h}]\end{array}$ & $\begin{array}{l}\text { Speed - cal. } \\
\text { dist. } 2[\mathrm{~km} / \mathrm{h}]\end{array}$ & $\begin{array}{l}\text { Acceleration in } \\
\mathrm{x} \text {-axis - cal. dist. } \\
1[\mathrm{~g}]\end{array}$ & $\begin{array}{l}\text { Acceleration in } \\
\text { x-axis - cal. dist. } \\
2[\mathrm{~g}]\end{array}$ & $\begin{array}{c}\text { Duration of } \\
\text { acceleration [s] }\end{array}$ \\
\hline Res F01 & 5.76 & 5.70 & 5.55 & 1.06 & 1.04 & 0.15 \\
\hline Res F02 & 8.67 & 8.06 & 8.50 & 1.65 & 1.74 & 0.108 \\
\hline Res R03 & 8.89 & 8.32 & 7.64 & 2.01 & 1.84 & 0.109 \\
\hline Res F04 & 6.67 & 6.27 & 6.46 & 1.40 & 1.44 & 0.084 \\
\hline Res F05 & 11.08 & 8.94 & 9.28 & 1.80 & 1.87 & 0.092 \\
\hline Res R06 & 10.14 & 9.83 & 10.31 & $3.05 / 2.12$ & $3.20 / 2.23$ & $0.075 / 0.125$ \\
\hline Rils F07 & 6.99 & 7.00 & 7.24 & 1.35 & 1.39 & 0.091 \\
\hline Rils F08 & 8.00 & 8.04 & 7.76 & 1.64 & 1.58 & 0.125 \\
\hline Rils R09 & 5.33 & 5.17 & 4.99 & $1.70 / 0.82$ & $1.64 / 0.80$ & $0.075 / 0.200$ \\
\hline Rils R10 & 10.14 & 8.87 & 8.30 & 3.09 & 2.89 & 0.059 \\
\hline
\end{tabular}

evaluating accelerations in the z-axis can arise due to time shift between peaks of acceleration in the $\mathrm{x}$-axis and in the $\mathrm{z}$-axis. Therefore, it is not correct to evaluate the maximum values of accelerations in particular axes only from the point where the maximum total acceleration was reached (which occurs mostly when the peak value of longitudinal acceleration was reached).

Monitoring units A1 and A2 placed in the height of buffers recorded high values of vertical acceleration mainly in impacts Res F01 (measured values were 0.80 and $1.36 \mathrm{~g}$ respectively), Res F02 (0.88 and $0.60 \mathrm{~g}$ respectively) and Res R06 (1.16 and $0.76 \mathrm{~g}$ respectively). High acceleration in the vertical axis was recorded also in impact Res F05 by monitoring units X1 and X2 (its peak value was 0.66 and $1.06 \mathrm{~g}$ respectively). Device MT6 in the middle of the wagon also detected strong vertical acceleration with two short-term peaks 1.70 and $1.47 \mathrm{~g}$ at this impact.

\section{Evaluation of parameters of impact from video analysis}

If the video analysis is used for evaluation of impact or braking parameters, there is important to take into account that correctness of acquired data depends on correct setting of calibration distance (referential distance according to which the software can count the video recording distances to real distances). It is also necessary to remove fish-eye effect. We used calibration distance of 1 meter, which was marked on the 
impacting wagon (Fig. 4). We also used referential point which was marked on the wagon and had contrastive colour. Tracker software using the function of Autotrack traced this referential point and recorded values of calculated speed and distance. The calibration distance is located in different place at the beginning and at the end of video recording and is recorded under different angle due to movement of observed wagon. So there are some differences in the measured values between using calibration distance in place at the beginning of video recording (cal. dist. 1, about $20 \mathrm{~m}$ before the place of impact) and calibration distance in the place at the end of recording (cal. dist. 2, at place of impact) due to distortion of video. Therefore, there are two data results. For purposes of comparison with other measuring equipment we used the average value of both results. Runs of speed and accelerations are similar when using both results (see Figs. 5 and 6 ), only calculated numerical values are different. Result values from video analysis are stated in Table 4.

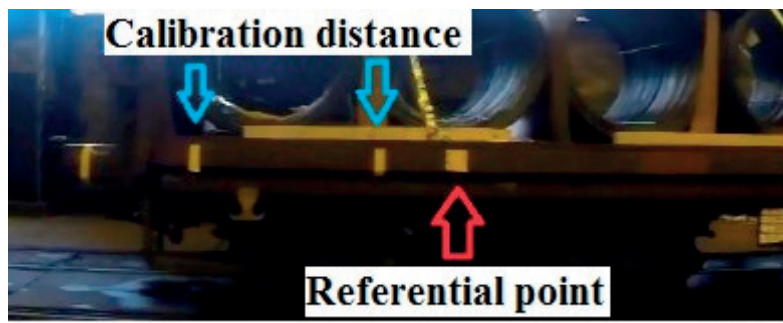

Fig. 4 Illustration of calibration distance and referential point on the wagon. Source: [3]

\section{Functionality of monitoring units during the impact tests}

The monitoring units which were used allow also recording the position and sending the automatic reports about detected accident situations. For purposes of these impact tests they were set to such initial level of acceleration for accident detection that all the impact tests should be evaluated as crashes. Monitoring unit A1 recorded all 10 impacts and 8 of them correctly evaluated and sent to the provider's information system. Some other smaller acceleration had occurred few seconds before both impacts which were not correctly evaluated. Unit A2 evaluated all 10 impacts as crashes, but in one case (Res F05) it also probably recorded other acceleration caused by an obstacle on the track which the wagon passed through. Unit X1 placed on the impact wagon recorded 9 of 10 observed impacts (only impact Rils R09 with low speed before impact missed) but only 5 of them were sent to the provider 's information system as crashes. Unit X2 recorded all 10 impacts and 7 of them evaluated as crashes. The impact tests were done at a closed hall, therefore the positions of impacts were not recorded very exact. Differences between determined positions and real positions of impacts were from 50 to $200 \mathrm{~m}$ in almost all the cases. Also the ride direction before the shock was in several cases recorded incorrectly or not recorded at all.

\section{Evaluation of runs of accelerations from device equipped with accelerometer and from video analysis}

In Figures 5 and 6 are stated runs of acceleration at two impacts as they were recorded by the device MT6 equipped with a tri-axial accelerometer and with video analysis of recordings from GoPro cameras. The time shift between peak values of longitudinal and vertical acceleration is visible in runs from MT6 device. Accessible data from monitoring units are not suitable for purpose of evaluating the run of acceleration, because they contain only the highest evaluated values and simplified runs of acceleration with interval of $0.1-0.3 \mathrm{~s}$, which means too small frequency for evaluating such short-term actions as wagon impact tests are. In both figures is visible that runs of acceleration acquired from the video analysis are very irregular.

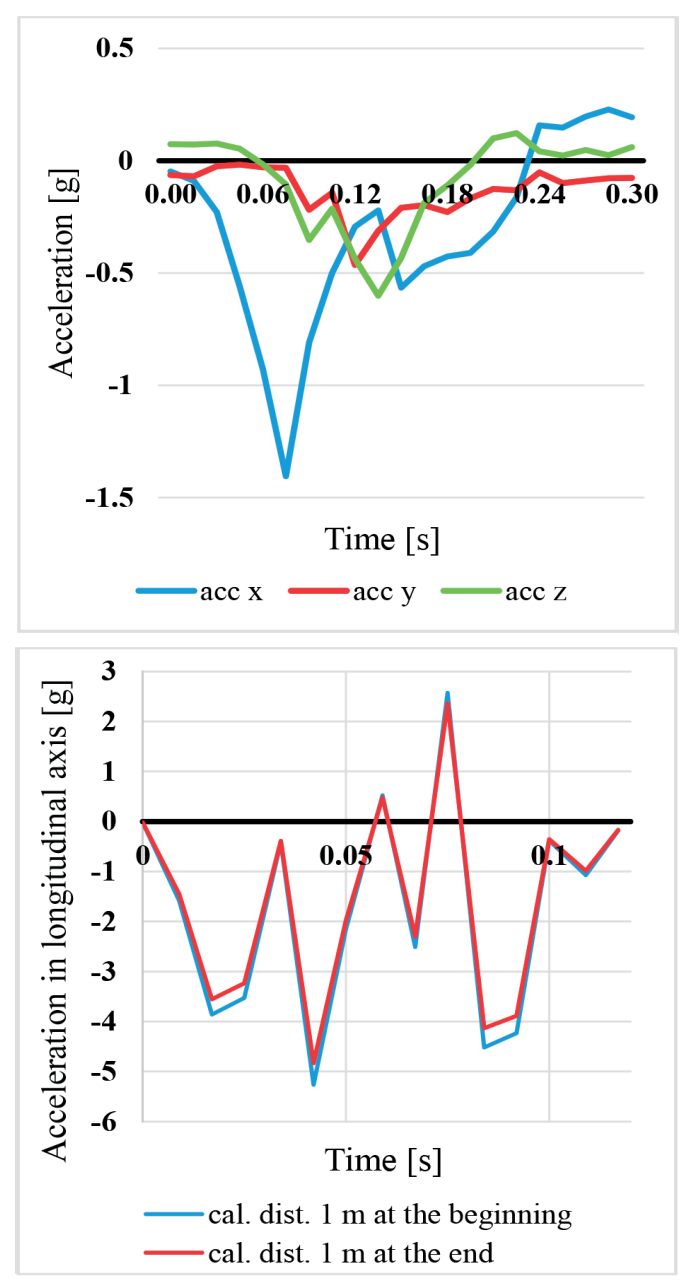

Fig. 5 Runs of accelerations in impact Res $R 03$ acquired from data from device MT6 (up) and from video analysis (down) 

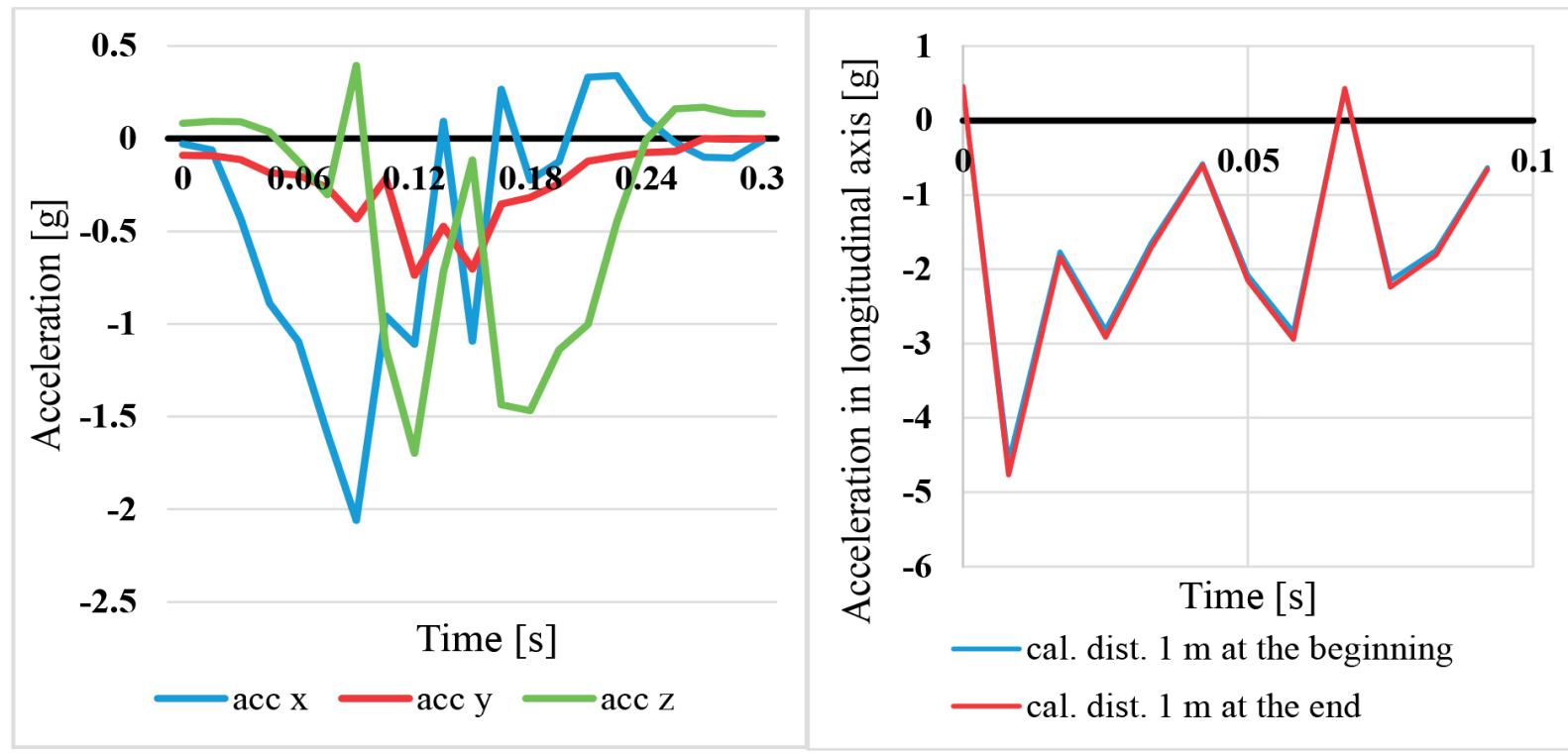

Fig. 6 Runs of accelerations in impact Res F05 acquired from data from device MT6 (on the left) and from video analysis (on the right)

\section{Conclusions}

In these impact tests the influence of wagon impacts on secured cargo consisting of coils of wire was observed. The securing of cargo only with the neighbouring coils caused that the cargo moved, the manner of securing used in the first series of impact tests was considered improper because the coil in the upper layer did not fulfil the crowding-out effect. Securing the coils in the upper layer to lashing points on the wagon can be considered as more proper way. Higher accelerations in the longitudinal axis were measured by measuring devices placed in the level of buffers than by devices placed higher on the wagon. Monitoring units intended for tracking and tracing of transport and detection of accidents were also used in these impact tests. The monitoring units did not reach such accuracy of position determination as it is usual when they are used on the roads or railways due to measuring in a closed building, probably not very well-covered with GPS signal. The majority of shocks during the impacts were detected correctly. However, not all shocks were evaluated as accidents including the accident message in provider's information system. The reliability of accident evaluation was about $75 \%$.

It is necessary to take into account that in some cases relatively high accelerations occur also in the vertical axis in commonly used wagons during shunting. These accelerations may reach about $1 \mathrm{~g}$. Such values of accelerations can be rarely reached also in the transverse axis. The construction of running gear and buffers of wagons strongly affects the accelerations actuating on cargo inside the wagons during shunting. Long stroke buffers capable to compress can rapidly reduce the accelerations actuating on cargo during impacts. Such buffers are capable to reduce the action of accelerations in the longitudinal axis on cargo even to $0.5-0.8 \mathrm{~g}$ for impacts at speed up to 10 $\mathrm{km} / \mathrm{h}$. When the speed before impact is higher, their dampening effect is lowered [2].

\section{References}

[1] International Union of Railways, RIV Appendix II, Loading Guidelines, Section 1, Principles, Paris, 2013

[2] ANDERSSON, P. et al.: Transport Quality on Railway Regarding Breakage, MariTerm AB: Hoganas, Sweden, 2006. Accessible at: http://www.mariterm.se/Download/BREAKAGE/ BREAKAGE.pdf

[3] MISKOV, M.: Possibilities of Using Software for Video Analysis in Selected Tests Related with Cargo Securing (in Slovak), Diploma thesis (consultant J. Jagelcak), University of Zilina, 2015. 